\title{
BMJ Open The Canadian National Vaccine Safety Network: surveillance of adverse events following immunisation among individuals immunised with the COVID-19 vaccine, a cohort study in Canada
}

Julie A Bettinger (D , ,,2 Manish Sadarangani, ${ }^{1,2}$ Gaston De Serres, ${ }^{3}$ Louis Valiquette, ${ }^{4}$ Otto G Vanderkooi, ${ }^{5,6}$ James D Kellner, ${ }^{5,6}$ Matthew P Muller, ${ }^{7,8}$ Karina A Top (D) , 9,10 Jennifer E Isenor, ${ }^{9,11}$ Allison McGeer, ${ }^{8,12}$ Kimberly Marty, ${ }^{1,2}$ Canadian Immunization Research Network

To cite: Bettinger JA, Sadarangani M, De Serres G, et al. The Canadian National Vaccine Safety Network: surveillance of adverse events following immunisation among individuals immunised with the COVID-19 vaccine, a cohort study in Canada. BMJ Open 2022;12:e051254. doi:10.1136/ bmjopen-2021-051254

- Prepublication history and additional supplemental material for this paper are available online. To view these files, please visit the journal online (http://dx.doi.org/10.1136/ bmjopen-2021-051254).

Received 13 March 2021 Accepted 17 December 2021

Check for updates

(C) Author(s) (or their employer(s)) 2022. Re-use permitted under CC BY-NC. No commercial re-use. See rights and permissions. Published by BMJ.

For numbered affiliations see end of article.

Correspondence to Dr Julie A Bettinger; jbettinger@bcchr.ubc.ca

\section{ABSTRACT}

Introduction COVID-19 vaccines require enhanced safety monitoring after emergency approval. The Canadian National Vaccine Safety Network monitors the safety of COVID-19 vaccines and provides enhanced monitoring for healthy, auto-immune, immunocompromised, pregnant and breastfeeding populations and allows for the detection of safety signals.

Methods and analysis Online participant reporting of health events in vaccinated and unvaccinated individuals 12 years of age and older is captured in three surveys: 1 week after dose 1, 1 week after dose 2 and 7 months after dose 1. Medically attended events are followed up by telephone. The number, percentage, rate per 10000 and incident rate ratios with $95 \%$ Cls are calculated by health event, vaccine type, sex and in 10-year age groups. Ethics and dissemination Each study site has Research Ethics Board approvals for the project (UBC Children's \& Women's, CIUSSS de I'Estrie-CHUS, Health PEI, Conjoint Health Research Ethics Board, University of Calgary and Alberta Health Services, IWK Health, Unity Health Toronto and CHU de Québec-Université Laval Research Ethics Boards). Individuals are invited to participate in this active surveillance and electronic consent is given before proceeding to each survey. Weekly reports are shared with public health and posted on the study website. At least one peer-reviewed manuscript is produced.

\section{INTRODUCTION}

The global pandemic of SARS-CoV-2 has caused $>100$ million cases and 2 million deaths globally. In response to this pandemic, an unprecedented effort to develop safe and effective vaccines was launched, and has led to the development of multiple vaccines demonstrated to be safe and effective in phase III trials. $^{1}$
Strengths and limitations of this study

- Captures COVID-19 vaccine safety outcomes in a more diverse population than that included in clinical trials.

- The inclusion of a control group of unvaccinated participants provides a similar comparator to the vaccinated group and allows for more rigorous analytic methods to detect potential safety signals.

- If a new event is detected, additional, enhanced follow-up can be done to inform public health action and policy.

- Potential exclusion of the most vulnerable as only those with an email address and fluent in French or English are eligible.

- Self-reporting of health events is subjective, may be subject to recall bias and limits the level of medical complexity that can be captured.

Controlling the pandemic requires public health mitigation measures and a global vaccine rollout that faces enormous challenges, not the least of which is widespread vaccine hesitancy in many countries. It is therefore of the utmost importance that regulatory and public health authorities monitor and transparently report all potential adverse events associated with each licensed vaccine in order to build and maintain public trust. ${ }^{2}$ Adverse events following immunisation (AEFI) are any "untoward medical occurrence which follows immunisation and which does not necessarily have a causal relationship with the usage of the vaccine'. ${ }^{3}$

The prelicensure trials of COVID-19 vaccines did not include sufficient numbers of 
individuals to identify rare adverse events (such as thrombosis with thrombocytopenia syndrome ${ }^{4}$ ) that occur with a frequency of 1-10 per 100000 or lower. ${ }^{5}$ Such events can only be detected when large numbers of people are given the vaccine and are followed for a sufficient period of time. ${ }^{6}$ Additionally, the prelicensure trials did not include sufficient numbers of older individuals, particularly the institutionalised elderly, or individuals with a variety of underlying health conditions and in most cases pregnant women and severely immunocompromised individuals were excluded from these trials entirely. ${ }^{7-9}$ Thus, active, postmarketing safety surveillance of COVID-19 vaccines is urgently needed.

One challenge with most postmarketing vaccine safety surveillance is that, unlike the clinical trials, it does not include a control group. ${ }^{10}$ This is an issue because not every health problem that occurs after receipt of a vaccine is caused by the vaccine. It is therefore important to include a control group that can track the background rate of health events in the community. This is particularly important in a pandemic, when some background events may be less common due to public health measures (eg, reduced incidence of seasonal respiratory viruses) while other health events may be related either to the pandemic virus itself, or to restricted access to healthcare. After vaccination, illness and/or health problems will happen as part of the background rate and only the excess above that rate should be attributed to the vaccine. To calculate the risks attributable to the vaccine, the background rate for common events needs to be determined in individuals who have not been recently vaccinated (control participants).

The Canadian National Vaccine Safety (CANVAS) network was designed to conduct active safety surveillance for pandemic vaccines, seasonal influenza vaccines and other new vaccines. CANVAS has been used to inform public and health authorities about the safety of seasonal influenza vaccines from 2010 to 2020, the H1N1 influenza vaccine in 2009 and the meningococcal B vaccine in 2014. ${ }^{11}{ }^{12}$ Additionally, CANVAS has routinely used a control group to help determine whether events occurring postvaccination represent true vaccine adverse events or are simply reflective of the background rates of illness. $^{12} 13$

In 2012, when the Agriflu vaccine was temporarily suspended in Canada (http://www.hc-sc.gc.ca/ahc-asc/ media/advisories-avis/_2012_164-eng.php), CANVAS was able to rapidly provide safety data on the vaccine to public health authorities to use in their evaluation of the product. In recent years, CANVAS has monitored the safety of seasonal influenza vaccines in over 50000 Canadians annually. ${ }^{12-14}$

In Canada, current vaccine pharmacovigilance involves both passive and active surveillance (monitoring) systems ${ }^{15}$ that are designed to detect, at minimal cost, very rare events in the large population of recipients of a broad range of vaccines. Passive surveillance, which involves spontaneous, voluntary reporting from multiple sources, suffers from under-reporting and reporting bias (based on age and severity). ${ }^{16} 17$ Active surveillance, which involves active searching for cases using standardised definitions by trained, paid nurses via the Canadian Immunization Monitoring Program, Active, occurs in children $(<17$ years of age) for specific conditions, such as seizures. ${ }^{18} 19$ Both types of monitoring can be slow to recognise safety signals and neither permits the calculation of population-based incidence rates of AEFIs. ${ }^{20}$ Finally, neither system is adequately designed to provide enhanced reporting for pandemic vaccines, with millions of doses administered over a short time period. ${ }^{21}$ The CANVAS surveillance network complements the ongoing passive and active surveillance systems by providing active safety monitoring for severe health events-events that prevent daily activities, work or school or require medical care-in vaccinated and unvaccinated individuals via case-cohort methods on a large number of individuals early in the COVID-19 vaccine campaign.

We therefore are conducting active vaccine surveillance for all COVID-19 vaccines approved for use in Canada and with the use of a control group to improve the ability to determine whether identified events are causally related to vaccination.

The main objectives of CANVAS are the following:

1. To identify common and uncommon health events associated with COVID-19 vaccination and to estimate their incidence by vaccine product, age, sex and other demographic and health-related factors.

2. To estimate in individuals vaccinated against COVID-19 the frequency of health events of sufficient severity to cause medical consultation, work absenteeism or prevent daily activities for each COVID-19 vaccine.

3. To determine whether severe health event rates for each COVID-19 vaccine are higher than severe health event rates in an unvaccinated control group.

\section{METHODS AND ANALYSIS \\ Study setting}

Participants are recruited from seven Canadian provinces and territories accounting for $>75 \%(34179760)^{22}$ of the Canadian population including Ontario, Quebec, British Columbia, Alberta, Nova Scotia, Yukon and Prince Edward Island.

\section{Recruitment vaccinated participants}

In Canada, healthcare is coordinated at the provincial or territorial level, and vaccine rollout plans differ regionally. For this reason, recruitment approaches vary at each site. However, three primary methods are used: (1) passive recruitment of COVID-19 vaccinated individuals at the time of vaccination via posters and public health information provided to vaccine recipients; (2) electronic invitation via the vaccination booking site when the vaccination appointment is made and (3) electronic invitation via email from contact information in the vaccine registry. 


\section{Controls}

We have four methods for inviting controls to participate: (1) existing CANVAS sites have previous CANVAS participants who have agreed to be contacted about research; (2) we are inviting CANImmunize (CANImmunize is a digital immunisation platform. CANImmunize and CANVAS have previously partnered to build and test a proof-of-concept stand-alone pilot app for CANVAS safety reporting. CANImmunize can identify account users (approximately 100000 ) by demographics and invite them to participate in the CANVAS-COVID control survey (www.canimmunize.ca) as controls; ${ }^{23}$ (3) publicity to direct individuals to our webpage www.CANVAS-COVID.ca and (4) volunteer research databases.

\section{Eligibility}

- Only individuals with an active email address are eligible to participate.

- For vaccinees: vaccinated with at least one dose of a COVID-19 vaccine in the previous 8 days.

- For controls: NOT vaccinated with any COVID-19 vaccine.

- French or English speaking.

- Individuals can contribute to both the control and vaccinated groups.

\section{Registration/Consent}

Online consent is collected for this study. Participants are given a description of the study prior to study enrolment. For those who wish to register, they may enrol at the study website (www.canvas-covid.ca) or via the link in the electronic invitation. First name, vaccination date, name of the vaccine received, vaccine lot number, email address and telephone number (s) are collected from participants.

\section{Study procedures}

Study participants (both vaccinated and unvaccinated) are asked to complete up to four online surveys. Vaccinated participants are sent an email containing the survey link 1 week after each vaccine dose and 7 months following the first dose inviting them to respond to a short survey. The survey is accessible by clicking on a link embedded in the email. The link provides secure access to the survey website using secure data transfer protocols. Telephone follow-up is done for those who report a medically attended event on any survey. If the participant fails to answer the survey, up to two automatic reminders are sent every 72 hours (figure 1).

The control survey collects data about the occurrence of health problems in the last 7 and 28 days and 6 months in unvaccinated participants. Adults who are expected to be vaccinated within the next 6 months, complete a retrospective control survey. All other controls are followed prospectively.

\section{Online data collection tool}

All sites use Research Electronic Data Capture (REDCap) software. $^{2425}$ REDCap is a secure, web-based software platform designed to support data capture for research studies, providing (1) an intuitive interface for validated data capture; (2) audit trails for tracking data manipulation and export procedures; (3) automated export procedures for seamless data downloads to common statistical packages and (4) procedures for data integration and interoperability with external sources. The version for this study is hosted and supported by the BC Children's Hospital Research Institute in Vancouver. The database is accessible through a secure web application with personal passwords for research staff, providing a secure environment (encryption, firewalls, frequent backups and recovery plan). For each survey, contact information

Figure 1 Study procedures.

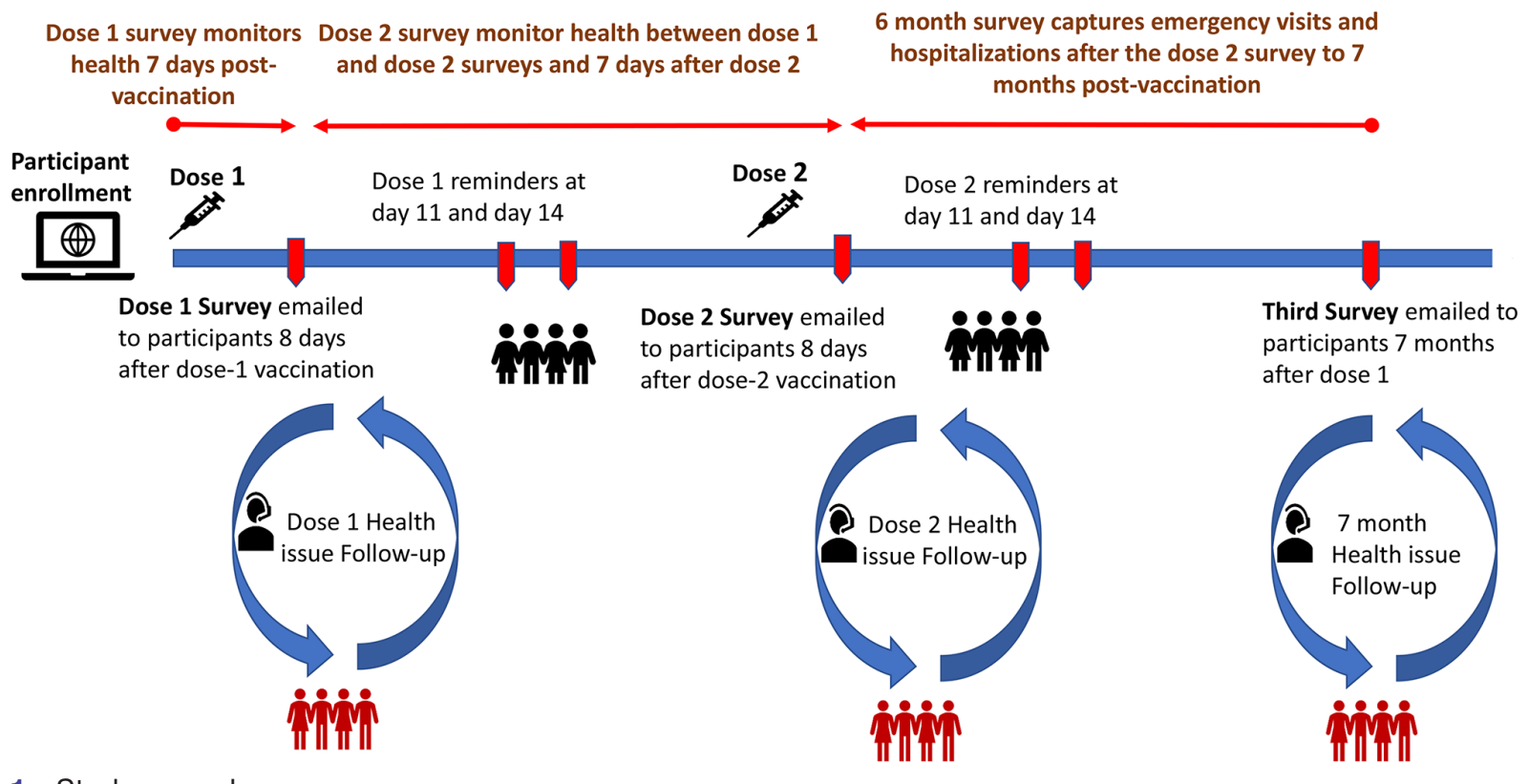


(email address, first name, telephone number) is stored in REDCap. Only research team members have access to participants' contact information. Survey data (without contact information) can be downloaded to the local hardware in Vancouver to allow for further and more complicated statistical analyses and modelling. Sites can receive de-identified copies of their site data via secure FTP transfer. Databases (including all contact information) are deleted from the REDcap server after data analysis is complete at the end of the study.

\section{Survey}

The dose 1 survey (sent 8 days after the first dose) contains the following questions: demographics data (participant age group in years, sex and gender); prior positive COVID-19 tests and disease severity; pregnancy and breastfeeding status; presence of auto-immune disease or immunocompromised; general health status (adapted from the Canadian Community Health Survey ${ }^{26}$ and the Clinical Frailty Scale ${ }^{27}$ and the occurrence or worsening of health events in the 7 days postvaccination. The dose 2 survey collects information on the occurrence or worsening of health events occurring between the dose 1 and dose 2 surveys and the occurrence or worsening of health events 8 days after the second vaccine dose. It collects the same demographic information as the dose 1 survey with an additional question on ethnicity. The 6-month survey collects information on the occurrence of emergency department visits and hospitalisations after the dose 1 survey up to 7 months postdose 1 vaccination.

\section{Outcomes}

Any new or worsening of an existing health event is determined by the question: in the first week ( 7 days) after your COVID-19 vaccine did you develop a new health problem or did an existing health problem get worse?

New or worsening of a severe health event is determined by the questions: was this health problem severe enough to prevent/stop normal activities; was this health problem severe enough to miss work/school; was this health problem severe enough to see a healthcare provider and did you see a healthcare provider for this health problem?

Emergency department visit or hospitalisation is determined by selecting emergency room or hospitalisation to the question: what type of medical visit did you have?

\section{Exposure variable}

Vaccination information is provided from the vaccination registry at three sites (BC and the two Quebec sites) or by the participant at four sites. Vaccine name, lot number and vaccination date are captured.

\section{Solicited symptoms}

On the dose 1 and dose 2 surveys, all participants are asked if they experienced an injection site reaction. Participants who indicate they experienced a severe event (prevented daily activities, work, school or required medical care) are then asked to select the symptoms they experienced from a list (see the dose 1 survey in the online supplemental materials. An 'other' category with a text field is provided for symptoms not included in the list. Then participants are provided with a pick list of the symptoms they indicated to select which symptom was the most severe. Onset and duration are captured for those with a severe health event. Diagnosis and treatment is captured for those with medically attended events. Hospitalisation date, duration and level of care is captured for those with hospitalisation.

\section{Non-responders}

A sample of participants (vaccinated and controls) who do not respond after the dose 2 and 6-month surveys may be followed up by telephone at each site. The nonresponder follow-up enables us to determine if there are differences in health event among those who complete the survey compared with those who do not. ${ }^{12}$ With participant consent, the missing surveys are completed with them at the time of the phone call and a set of questions are asked about why they did not complete the survey online. We seek to follow-up with approximately $2 \%$ of non-responders, depending on the total number of participants, total number of non-responders and available study budget.

\section{Event follow-up}

Vaccinated participants reporting adverse events severe enough to seek medical attention are contacted by a staff member specifically trained in obtaining a history of possible adverse events following immunisation. As several AEFI can be reported by an individual, the call also serves to clarify the main AEFI that triggered the medical consultation. These events may be reportable to public health as required by local public health guidelines. Controls reporting health events severe enough to seek medical attention also are contacted. Follow-up calls are made ideally within 48-72 hours of the event report. Where possible, Brighton Collaboration definitions are used to define events. ${ }^{5}$ However, given the self-reported nature of this study, sufficient medical detail is frequently not available from the participant to enable classification of the event according to Brighton definitions.

Once validated, the health event follow-up data are entered into the database, allowing near real-time data analysis. Participants remain identified by a unique number unless the event is reportable to public health. ${ }^{28-32}$

\section{Signal detection response}

Should a new AEFI or safety signal be detected, either through CANVAS or other surveillance systems, participants may be asked to respond to an additional survey in order to better define and quantify the event. Administration of this survey would follow the same procedures as described for above surveys, but would ask specific questions about the identified event.

\section{Sample size}

For each vaccine product, the target sample size is 240 000 vaccinated participants and 50000 controls. Across all age groups, the above sample size would allow us to 
detect health events with a relative risk of 1.14 (for events occurring at a $1 \%$ background rate) compared with controls and importantly, to conduct adequately powered subgroup analyses by age and vaccine product. The 240 000 sample per product allows for a sample of $\sim 34000$ for each of the seven 10-year adult age groups. Within each 10-year age group, a sample of 34000 allows detection of a difference in risk ratios of 1.4 with $80 \%$ power and a two-sided significance level of $95 \%$. A total of 27639 802 adults 18 years of age and older number are eligible for vaccine. ${ }^{33}$ The sample will cover approximately $3 \%$ of eligible Canadians (3 vaccine products at 240000 each $=720000 / 27639802$ ).

The control group sample size was strictly based on the maximum sample we thought possible given our recruitment via existing participants and CANImmunize. If we are able to publicise the study to generate interest and use other research databases to invite control participants, we may be able to increase the control group size. We are monitoring specific age groups as enrolment occurs, in both the vaccinated and control groups and may conduct more targeted recruitment if needed and/or restrict enrolment when specific sample size age categories have been met.

\section{Analytic plan}

The main objective of this project is to estimate in adults vaccinated against COVID-19 and those not vaccinated (the control group) the frequency of severe health events (ie, events of sufficient severity to cause work absenteeism, medical consultation or prevent daily activities) by vaccine type and age group.

For each vaccine type and the unvaccinated group, the number, proportion and rate per 10000 is calculated for any events, severe events and emergency department/ hospitalisations after dose 1 and after dose 2. Participants who receive a mixed vaccine schedule are examined as a separate vaccine type after dose 2 . Incident rate ratios by vaccine type with $95 \%$ CIs for each outcome are calculated. Multivariable Poisson regression is used to estimate relative risk of any and severe events following each dose of each vaccine type overall and by age group adjusted for province. Models will be examined for sex and gender differences.

The secondary objectives are to explore the incidence of severe events by auto-immune, immunocompromised, pregnancy and breastfeeding status and prior COVID-19 infection.

Frequencies and proportions are calculated for any events, severe events and emergency department/hospitalisations after dose 1 and after dose 2 and in the unvaccinated group by auto-immune, immunocompromised, pregnancy and breastfeeding status and prior COVID-19 infection. Multivariable Poisson regression is used to estimate relative risk of any severe events following exposure of COVID-19 vaccination in the auto-immune, immunocompromised, pregnancy and breastfeeding groups after controlling for age, sex, gender, health status, province, prior COVID infection and vaccine interval. Several Poisson regression models are used for the different outcome variables. If the baseline characteristics between the vaccinated and unvaccinated groups are not comparable, propensity score analysis will be considered to achieve exchangeability between groups.

Multivariable logistic regression is used for the prior COVID infection analysis to determine the difference in risk for a severe event or emergency department/hospitalisation at each dose among vaccinated participants with and without prior COVID-19 infections after controlling for age, sex, gender, health status and province. Several logistic regression models are used for the different outcome variables and different time points.

\section{ETHICS AND DISSEMINATION}

Each study site has Research Ethics Board approvals for the project (UBC Children's \& Women's, CIUSSS de l'Estrie-CHUS, Health PEI, Conjoint Health Research Ethics Board, University of Calgary and Alberta Health Services, IWK Health, Unity Health Toronto and CHU de Québec-Université Laval). Individuals are invited to participate in this active surveillance and electronic consent is given before proceeding to each survey.

\section{Author affiliations}

${ }^{1}$ Vaccine Evaluation Center, BC Children's Hospital Research Institute, Vancouver, British Columbia, Canada

${ }^{2}$ Pediatrics, University of British Columbia, Vancouver, British Columbia, Canada ${ }^{3} \mathrm{CHU}$ de Québec-Université Laval, Quebec, Québec, Canada

${ }^{4}$ Centre Intégré Universitaire de Santé et de Services Sociaux de l'Estrie-Centre Hospitalier Universitaire de Sherbrooke, Sherbrooke, Quebec, Canada

${ }^{5}$ Alberta Children's Hospital Research Institute, Calgary, Alberta, Canada

${ }^{6}$ Pediatrics, University of Calgary, Calgary, Alberta, Canada

${ }^{7}$ Medicine, Unity Health Toronto, Toronto, Ontario, Canada

${ }^{8}$ University of Toronto, Toronto, Ontario, Canada

${ }^{9}$ Canadian Center for Vaccinology, Dalhousie University, Halifax, Nova Scotia, Canada

${ }^{10}$ Pediatrics, IWK Health Centre, Halifax, Nova Scotia, Canada

${ }^{11}$ College of Pharmacy, Dalhousie University, Halifax, Nova Scotia, Canada

${ }^{12}$ Sinai Health System, Toronto, Ontario, Canada

Collaborators Canadian Immunization Research Network.

Contributors JAB, GDS, MPM, JEl, KM, MS, JDK, AMCG, OGV, LV and KAT contributed to the design of the study and the collection of data. JAB wrote and finalised the protocol. GDS, MPM, JEI, KM, MS, JDK, AMcG, OGV, LV and KAT read and revised and approved the protocol.

Funding This work was supported by the COVID-19 Vaccine Readiness funding from the Canadian Institutes of Health Research and the Public Health Agency of Canada CANVAS grant number CVV-450980. This project was supported by funding from the Public Health Agency of Canada, through the Vaccine Surveillance Reference group and the COVID-19 Immunity Task Force. MS is supported via salary awards from the BC Children's Hospital Foundation, the Canadian Child Health Clinician Scientist Programme and the Michael Smith Foundation for Health Research.

Competing interests JAB, MPM, JDK, AMcG, JEl and KM have no competing interests. MS has been an investigator on projects funded by GlaxoSmithKline, Merck, Pfizer, Sanofi-Pasteur, Seqirus, Symvivo and VBI Vaccines outside the submitted work. All funds have been paid to his institute, and he has not received any personal payments. GDS and LV report grants from Pfizer outside the submitted work. KAT reports consultancy fees from Pfizer and a grant from GSK outside the submitted work. OGV has been an investigator, coinvestigator and/or expert 
panellist on projects funded by GlaxoSmithKline, Merck, Pfizer and Seqirus, outside the submitted work.

\section{Patient consent for publication Not applicable.}

Provenance and peer review Not commissioned; externally peer reviewed.

Supplemental material This content has been supplied by the author(s). It has not been vetted by BMJ Publishing Group Limited (BMJ) and may not have been peer-reviewed. Any opinions or recommendations discussed are solely those of the author(s) and are not endorsed by BMJ. BMJ disclaims all liability and responsibility arising from any reliance placed on the content. Where the content includes any translated material, BMJ does not warrant the accuracy and reliability of the translations (including but not limited to local regulations, clinical guidelines, terminology, drug names and drug dosages), and is not responsible for any error and/or omissions arising from translation and adaptation or otherwise.

Open access This is an open access article distributed in accordance with the Creative Commons Attribution Non Commercial (CC BY-NC 4.0) license, which permits others to distribute, remix, adapt, build upon this work non-commercially, and license their derivative works on different terms, provided the original work is properly cited, appropriate credit is given, any changes made indicated, and the use is non-commercial. See: http://creativecommons.org/licenses/by-nc/4.0/.

ORCID iDs

Julie A Bettinger http://orcid.org/0000-0002-2118-4174

Karina A Top http://orcid.org/0000-0002-9981-368X

\section{REFERENCES}

1 Krammer F. SARS-CoV-2 vaccines in development. Nature 2020;586:516-27.

2 World Health Organization. Covid-19 vaccines: safety surveillance manual. Geneva: World Health Organization, 2020.

3 Council for International Organizations of Medical Sciences World Health Organization (WHO). Definition and application of terms for vaccine pharmacovigilance: report of CIOMS/WHO Working group on vaccine pharmacovigilance. Geneva: Council for International Organizations of Medical Sciences World Health Organization (WHO), 2012.

4 Chen R, Black S. Updated proposed Brighton collaboration process for developing a standard case definition for study of new clinical syndrome $\mathrm{X}$, as applied to thrombosis with thrombocytopenia syndrome (tTS) Atlanta, 2021. Available: https://brightoncollabor ation.us/wp-content/uploads/2021/05/TTS-Interim-Case-Definitionv10.16.3-May-23-2021.pdf [Accessed 20 Aug 2021].

5 Bonhoeffer J, Bentsi-Enchill A, Chen RT, et al. Guidelines for collection, analysis and presentation of vaccine safety data in surveillance systems. Vaccine 2009;27:2289-97.

6 Fritzell B. Detection of adverse events: what are the current sensitivity limits during clinical development? Vaccine 2001;20:S47-8.

7 Polack FP, Thomas SJ, Kitchin N, et al. Safety and efficacy of the BNT162b2 mRNA Covid-19 vaccine. N Engl J Med 2020;383:2603-15

8 Baden LR, El Sahly HM, Essink B, et al. Efficacy and safety of the mRNA-1273 SARS-CoV-2 vaccine. N Engl J Med 2021;384:403-16.

9 Voysey M, Clemens SAC, Madhi SA, et al. Safety and efficacy of the ChAdOx1 nCoV-19 vaccine (AZD1222) against SARS-CoV-2: an interim analysis of four randomised controlled trials in Brazil, South Africa, and the UK. Lancet 2021;397:99-111.

10 Chen RT, Glasser JW, Rhodes PH, et al. Vaccine safety Datalink project: a new tool for improving vaccine safety monitoring in the United States. The vaccine safety Datalink team. Pediatrics 1997:99:765-73.

11 De Serres G, Gariépy M-C, Coleman B, et al. Short and long-term safety of the 2009 AS03-adjuvanted pandemic vaccine. PLoS One 2012; $7:$ e38563.
12 Bettinger JA, Rouleau I, Gariepy MC, et al. Successful methodology for large-scale surveillance of severe events following influenza vaccination in Canada, 2011 and 2012. Euro Surveill 2015;20:21189.

13 Bettinger JA, De Serres G, Valiquette L, et al. 2017/18 and 2018/19 seasonal influenza vaccine safety surveillance, Canadian national vaccine safety (canvas) network. Eurosurveillance2020;25.

14 Ahmed MA, Naus M, Singer J, et al. Investigating the association of receipt of seasonal influenza vaccine with occurrence of anesthesia/ paresthesia and severe headaches, Canada 2012/13-2016/17, the Canadian vaccine safety network. Vaccine 2020;38:3582-90.

15 Public Health Agency of Canada. Part 2 vaccine safety and adverse events following immunization. Canadian immunization guide. evergreen edition ED. Public Health Agency of Canada, 2012.

16 National passive surveillance Geneva: World Health organization, 2019. Available: https://www.who.int/immunization/monitoring surveillance/burden/vpd/surveillance_type/passive/en/ [Accessed 23 Apr 2019].

17 Varricchio F, Iskander J, Destefano F, et al. Understanding vaccine safety information from the vaccine adverse event reporting system. Pediatr Infect Dis J 2004;23:287-94.

18 Scheifele DW. Impact after 17 years: lessons learned about successful networking. Paediatr Child Health 2009;14:33-5.

19 Scheifele DW, Halperin SA, Program IM. Active: a model of active surveillance of vaccine safety. Semin Pediatr Infect Dis 2003;14:213-9.

20 Iskander J, Broder K. Monitoring the safety of annual and pandemic influenza vaccines: lessons from the US experience. Expert Rev Vaccines 2008;7:75-82.

21 Gold MS, Effler P, Kelly H, et al. Febrile convulsions after 2010 seasonal trivalent influenza vaccine: implications for vaccine safety surveillance in Australia. Med J Aust 2010;193:492-3.

22 Table 17-10-0009-01 population estimates, quarterly: statistics Canada, 2021. Available: https://doi.org/10.25318/1710000901-eng

23 Wilson K, Atkinson KM, Westeinde J, et al. An evaluation of the feasibility and usability of a proof of concept mobile APP for adverse event reporting post influenza vaccination. Hum Vaccin Immunother 2016;12:1-11.

24 Harris PA, Taylor R, Thielke R, et al. Research electronic data capture (REDCap)--a metadata-driven methodology and workflow process for providing translational research informatics support. J Biomed Inform 2009;42:377-81.

25 Harris PA, Taylor R, Minor BL, et al. The REDCap Consortium: building an international community of software platform partners. $J$ Biomed Inform 2019;95:103208.

26 Canadian community Health Survey (CCHS) - annual component 2020: Statistics Canada, 2020. Available: https://www23.statcan.gc. $\mathrm{ca} / \mathrm{imdb} / \mathrm{p} 3$ Instr.pl?Function=assemblelnstr\&a=1\&\&lang=en\&Item Id=1262397 [Accessed 18 Dec 2020].

27 Rockwood K, Song X, MacKnight C, et al. A global clinical measure of fitness and frailty in elderly people. CMAJ 2005;173:489-95.

28 COVID-19 vaccine information for health care providers Charlottetown: government of Prince Edward island, 2021. Available: https://www.princeedwardisland.ca/en/information/health-andwellness/covid-19-vaccine-information-for-health-care-providers

29 It's the Law: Report Adverse Events Following Immunization (AEFI) Halifax: Nova Scotia Health, 2021. Available: https://www. novascotia.ca/dhw/cdpc/documents/13087_AdverseEventsPoster En.pdf [Accessed 20 Aug 2021].

30 Vaccine safety Toronto: public health Ontario, 2021. Available: https://www.publichealthontario.ca/en/health-topics/immunization/ vaccine-safety [Accessed 20 Aug 2021].

31 Adverse event following immunization (AEFI) reporting for all health care practitioners in Alberta Edmonton: Alberta health services, 2021 Available: https://www.albertahealthservices.ca/info/Page16187.aspx [Accessed 18 Aug 2021].

32 Adverse event following immunization Vancouver: bc center for disease control, 2021. Available: http://www.bccdc.ca/healthprofessionals/clinical-resources/adverse-events-followingimmunization [Accessed 20 Aug 2021].

33 Table 17-10-0005-01 population estimates on July 1st, by age and sex: statistics Canada, 2021. Available: https://doi.org/10.25318/ 1710000501-eng. 